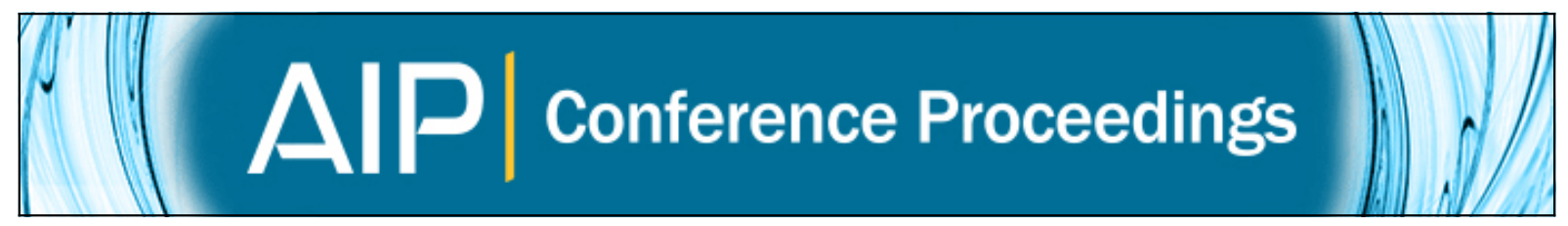

\title{
Cyclotron Resonance near the Charge Neutrality Point of Graphene
}

Zhigang Jiang, E. A. Henriksen, P. Cadden-Zimansky, L.-C. Tung, Y.-J. Wang, P. Kim, and H. L. Stormer

Citation: AIP Conference Proceedings 1399, 773 (2011); doi: 10.1063/1.3666605

View online: http://dx.doi.org/10.1063/1.3666605

View Table of Contents: http://scitation.aip.org/content/aip/proceeding/aipcp/1399?ver=pdfcov

Published by the AIP Publishing

\section{Articles you may be interested in}

Competitive interfacial charge transfer to graphene from the electrode contacts and surface adsorbates Appl. Phys. Lett. 106, 083107 (2015); 10.1063/1.4913669

Sample-charged mode scanning polarization force microscopy for characterizing reduced graphene oxide sheets J. Appl. Phys. 115, 244302 (2014); 10.1063/1.4883538

Charge transport mechanisms of graphene/semiconductor Schottky barriers: A theoretical and experimental study

J. Appl. Phys. 115, 013701 (2014); 10.1063/1.4859500

Charge transfer between reduced graphene oxide sheets on insulating substrates

Appl. Phys. Lett. 103, 053107 (2013); 10.1063/1.4817252

First-principles investigation of charge density analysis of various adatom adsorptions on graphene AIP Conf. Proc. 1399, 793 (2011); 10.1063/1.3666615 


\title{
Cyclotron Resonance near the Charge Neutrality Point of Graphene
}

\author{
Zhigang Jiang $^{1}$, E. A. Henriksen ${ }^{2}$, P. Cadden-Zimansky ${ }^{3,4}$, L.-C. Tung $^{4}$, Y.-J. \\ Wang $^{4}$, P. Kim ${ }^{3}$, and H. L. Stormer ${ }^{3,5}$ \\ ${ }^{I}$ School of Physics, Georgia Institute of Technology, Atlanta, Georgia, U.S.A. \\ ${ }^{2}$ Department of Physics, California Institute of Technology, Pasadena, California, U.S.A. \\ ${ }^{3}$ Department of Physics and Applied Physics, Columbia University, New York, New York, U.S.A. \\ ${ }^{4}$ National High Magnetic Field Laboratory, Tallahassee, Florida, U.S.A. \\ ${ }^{5}$ Bell Laboratories, Alcatel-Lucent, Murray Hill, New Jersey, U.S.A.
}

\begin{abstract}
We perform infrared studies of the Landau level (LL) transitions in monolayer graphene in magnetic fields up to $B=18 \mathrm{~T}$. We observe both intra-band transitions between neighboring hole/electron LLs, as well as inter-band transitions between hole and electron LLs, all proportional to $\sqrt{B}$ in energy. The lack of precise scaling between different LL transitions and the unexpectedly large energy shifts as a function of the LL filling factor near the charge neutrality point suggest considerable contributions of many-body effects to the infrared transition energies.
\end{abstract}

Keywords: Graphene, cyclotron resonance, and many-body effects.

PACS: 78.66.Tr; 71.70.Di; 76.40.+b

\section{INTRODUCTION}

In a perpendicular magnetic field, $B$, the motions of the charge carriers in graphene quantize into Landau levels (LLs), with an unusual energy dependence on both $B$ and the LL index, $n$, described in a singleparticle model by [1]

$$
E_{n}=\operatorname{sgn}(n) \sqrt{2|n|} \hbar \tilde{c} / l_{B} .
$$

Here $\hbar$ is reduced Planck's constant, $\tilde{c} \approx 10^{6} \mathrm{~m} / \mathrm{s}$ the band velocity, $l_{B}=\sqrt{\hbar /(e B)}$ the magnetic length, and $e$ the electron charge. The index $n$ runs over both positive and negative integers, representing electron and hole-like states, and includes a field-independent $n=0 \mathrm{LL}$ which is located at zero energy (the charge neutrality point).

\section{EVIDENCE OF MANY-BODY EFFECTS PROBED BY INFRARED LANDAU LEVEL SPECTROSCOPY}

In conventional two-dimensional systems with parabolic dispersions, Kohn's theorem [2] explains that e-e interactions will have no impact on the LL transition energies observed in infrared (IR) measurements of cyclotron resonance (CR). Instead, an observed resonance energy coincides with its noninteracting value, provided that the system is translationally invariant, which, in spite of residual disorder, can usually be assumed to hold. Yet, Kohn's theorem fails in the case of graphene, where the linear dispersion may be viewed as a case of extreme nonparabolicity, and thus many-body effects are expected to contribute to the LL transition energies. Here we provide experimental evidence for many-body effects in the graphene $\mathrm{CR}$ from three different points of view:

(1) The lack of precise scaling between the lowest intra-band LL transition and the lowest inter-band transition [3]. The insets to Fig. 1 show the $n=-1 \rightarrow$ 0 intra-band transition $\left(\mathrm{T}_{1}\right.$, blue $)$ and the $n=-2 \rightarrow$ +1 and $n=-1 \rightarrow+2$ inter-band transitions $\left(\mathrm{T}_{2}\right.$, red $)$ measured at $18 \mathrm{~T}$. The transition energies are proportional to $\sqrt{B}$, as expected for graphene, and the deduced band velocity is $\tilde{c} \approx 1.1 \times 10^{6} \mathrm{~m} / \mathrm{s}$. In the single-particle picture of Eq. (1), the $T_{1}: T_{2}$ energy ratio scales as $1:(\sqrt{2}+1)$. However, as indicated in the main panel of Fig. 1, we observe a clear deviation from the $1:(\sqrt{2}+1)$ ratio, which sheds doubt on the applicability of Eq. (1) in fully interpreting the IR data 


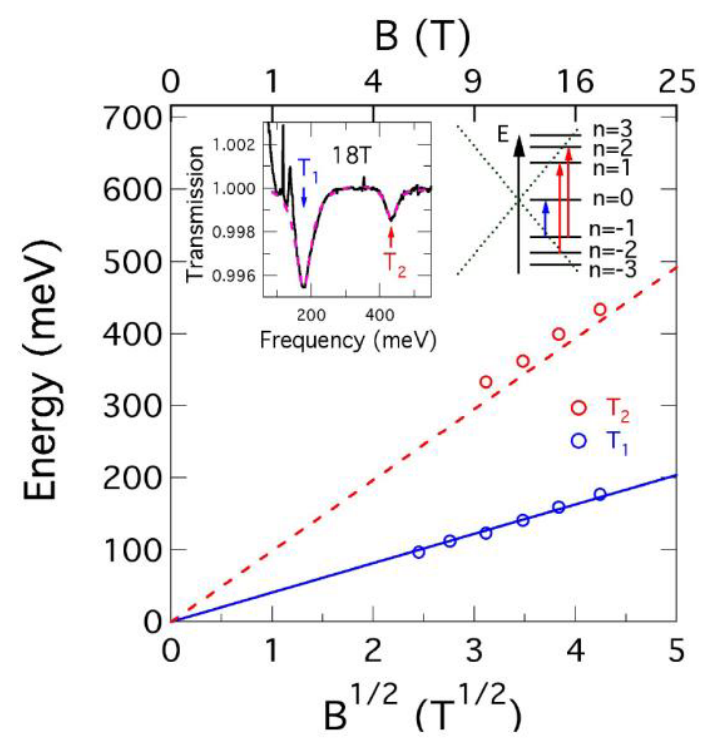

FIGURE 1. $B$-field dependence of the resonance energies in graphene. Solid blue line is a best $\sqrt{B}$-fit to the $\mathrm{T}_{1}$ (blue) transition, while the dashed red line represents a scaling of the solid line by a factor $(\sqrt{2}+1)$. Left inset, a representative transmission spectrum at $B=18 \mathrm{~T}$; right inset, schematic of the corresponding LL transitions.

on graphene. Indeed, many-body corrections to the bare LL transitions have recently been calculated in the literature. Iyengar et al [4] arrive at quite large many-body contributions, $\sim 60 \mathrm{meV}$ at $B=18 \mathrm{~T}$ with an assumed dielectric constant of $\varepsilon=4$ and with the same sign as the observed deviation.

(2) The lack of precise scaling between the low intra-band LL transitions near the charge neutrality point and the high LL transitions [5]. Specifically, we map out the intra-band LL transitions between two neighboring hole or electron LLs of monolayer graphene, up to $n=7$. As expected, the corresponding energies of all observed transitions are proportional to $\sqrt{B}$, enabling us to extract a band velocity for each transition. We find the deduced band velocity is $1.12-1.13 \times 10^{6} \mathrm{~m} / \mathrm{s}$ near charge neutrality (zero energy), while it is considerably smaller $0.96-1.0 \times$ $10^{6} \mathrm{~m} / \mathrm{s}$ at higher energies. This observation is consistent with a band velocity renormalization picture resulting from e-e interactions [6], and in agreement with other reported works using different measurement techniques $[7,8]$.

(3) The unexpected large energy shifts as a function of the LL filling factor, $v$, near the charge neutrality point [9]. Figure 2 shows the CR data for $v= \pm 2$ and 0 in a high mobility monolayer graphene sample at $B=18 \mathrm{~T}$. The transitions responsible for the two resonance peaks in each trace are illustrated schematically in the upper left inset to Fig. 3. The inter-band peak (at $440 \mathrm{meV}$ ) shifts by less than $1 \%$ as $v$ is changed. In contrast, the lower energy peaks (at

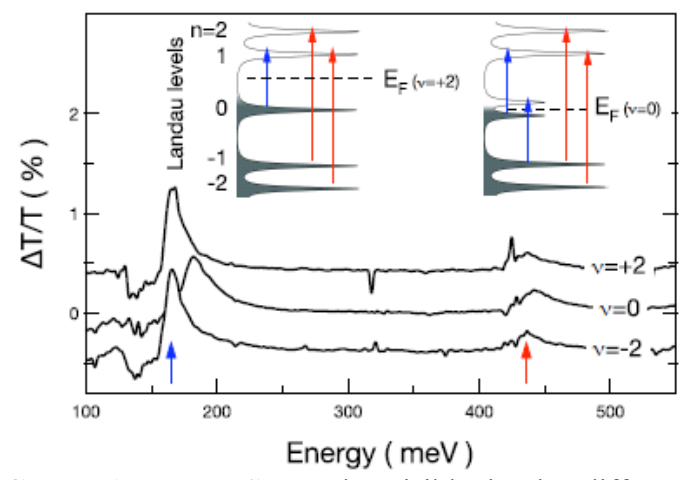

FIGURE 2. Two CR peaks visible in the differential transmission $(\Delta T / T)$ traces at $B=18 \mathrm{~T}$. The underlying transitions are $n=0 \rightarrow+1$ and/or $n=-1 \rightarrow 0$ near 170 meV (blue arrows), and $n=-1 \rightarrow+2$ and $n=-2 \rightarrow+1$ at $440 \mathrm{meV}$ (red arrows). Traces are offset for clarity. Left inset: LL schematic showing transitions at $v=+2$, when $E_{F}$ lies between the zeroth and first LLs. Right inset: proposed LL schematic at charge neutrality $(v=0)$, showing a gap in the $n=0 \mathrm{LL}$ which would shift the lowest energy CR transitions. (Reproduced from Ref. [9]).

$165-180 \mathrm{meV})$ show a strong upshift $(\sim 8 \%)$ in energy at $v=0$. The magnitude of this shift, and its magnetic field dependence, suggests that an interactionenhanced energy gap opens in the $n=0$ level at high magnetic fields, as shown schematically in the upper right inset to Fig. 3. Thus, at charge neutrality the energy of the lower (blue) CR transition is increased by half of the size of the gap, while the inter-band transition (red) energy is intact. Our CR shift gives rise to a gap size of $30 \mathrm{meV}$ at $B=18 \mathrm{~T}$, much larger than the bare Zeeman energy splitting. Note that lower mobility samples have consistently smaller energy shifts at $v=0$, a further indication that they originate from many-body effects.

\section{ACKNOWLEDGMENTS}

This work is supported by the DOE (DE-FG0207ER46451). A portion of this work was performed at the National High Magnetic Field Laboratory, which is supported by NSF Cooperative Agreement No. DMR0654118 , by the State of Florida, and by the DOE.

\section{REFERENCES}

1. J. W. McClure, Phys. Rev. 104, 666 (1956).

2. W. Kohn, Phys. Rev. 123, 1242 (1961).

3. Z. Jiang et al, Phys. Rev. Lett. 99, 106802 (2007).

4. A. Iyengar et al, Phys. Rev. B 75, 125430 (2007).

5. Z. Jiang et al, (unpublished).

6. Y. A. Bychkov et al, Phys. Rev. B 77, 125417 (2008).

7. Z. Q. Li et al, Nat. Phys. 4, 532 (2008).

8. M. B. Lundeberg et al, Nat. Phys. 5, 894 (2009).

9. E. A. Henriksen et al, Phys. Rev. Lett. 104, 067404 (2010). 